\title{
Moving Multiple Curves/Surfaces Approximation of Mixed Point Clouds
}

\author{
Wenyue Feng · Zhouwang Yang • Jiansong Deng
}

Received: 12 April 2014 / Revised: 29 May 2014 / Accepted: 7 June 2014 /

Published online: 19 July 2014

(C) School of Mathematical Sciences, University of Science and Technology of China and

Springer-Verlag Berlin Heidelberg 2014

\begin{abstract}
We propose a local model called moving multiple curves/surfaces approximation to separate mixed scanning points received from a thin-wall object, where data from two sides of the object may be mixed due to measurement error. The cases of two curves (including plane curves and space curves) and two surfaces in one model are mainly elaborated, and a lot of examples are tested.
\end{abstract}

Keywords Moving multiple curves/Surfaces approximation · Mixed point cloud · Constrained optimization $\cdot$ Surface fitting

Mathematical Subject Classification (2010) 26B05 $\cdot 41 \mathrm{~A} 29 \cdot 65 \mathrm{P} 10$

\section{Introduction}

Nowadays, with the development of scanning technique, obtaining scanned point cloud data via scanner becomes more and more convenient. However, because of the limitation of measurement equipment, measurement technology, and the measurement process, the acquired points usually deviate from their true positions, even mix together, when the points come from multiple close surfaces such as thin machine parts. But it is still possible to get the underlying smooth shapes (curves or surfaces) from the unorganized noisy point cloud. So modeling from noisy data is receiving a growing amount of attention.

The reconstructed surface from unorganized points can be represented in two ways. One method is analytic surfaces obtained by conventional least squares (LS) method,

W. Feng $\cdot$ Z. Yang $\cdot$ J. Deng $(\varangle)$

School of Mathematical Sciences, University of Science and Technology of China, Hefei 230026, China

e-mail: dengjs@ustc.edu.cn

W. Feng

e-mail: fengwy@mail.ustc.edu.cn 
and another is point set surfaces [3]. The size of a point set in point-based representation should be as small as possible, in the sense that the point set is neither noisy nor redundant. It can be obtained by the moving least squares (MLS) method. The MLS method was proposed by Lancaster and Salkauskas [14] for smoothing and interpolating scattered data. The idea is to start with a weighted least squares (WLS) formulation [18] for an arbitrary fixed point in $R^{d}$ and then move this point over the entire parameter domain, where a WLS fit is computed and evaluated for each point, respectively. In this paper, we combine the LS method with a point-based representation to do curve and surface fitting.

As an extension of the technique of MLS, moving parabolic approximation (MPA) [27] is based on some given assumptions. By MPA, an improved point-based model of curve or surface is reconstructed, while also the differential properties of the underlying smooth manifold are estimated. The MPA models are solved by optimization algorithms. MLS and MPA are effective to deal with a point cloud from a single curve or surface. However, when the scanned points are from multiple close curves or surfaces, they may mix together, and we cannot distinguish which point belongs to which curve or surface, especially in the central area. This usually occurs when scanning points from the patches of broken relics [12], thin machine parts and other thin-wall objects. Motivated by MLS and MPA, we provide a model called moving multiple curves/surfaces approximation (MMC/SA) to separate mixed data and recover the underlying shapes. Instead of two boundaries approximation, shown in Fig. 1a, our work is effective in the situation depicted in Fig. 1b. For MMC/SA, it is not necessary to investigate which point belongs to which curve or which surface beforehand.

The rest of this paper is organized as follows. Section 2 reviews the related work briefly. Section 3 introduces the MMC/SA and develops two straight lines and two parabolas approximation for two close plane curves. Additionally, Sect. 3 introduces two planes and two paraboloids approximation for two close surfaces and sets up four appropriate optimization models. Section 4 shows the implementation of the algorithm. Section 5 presents some examples and discussions and lastly, Sect. 6 concludes the paper.

\section{Related Work}

Since this paper is in connection with MLS and MPA, we will review the relevant work next.

Fig. 1 a Two boundaries approximation; b two curves obtained using the results of MMCA models according to the same red points as (a). (Color figure online)

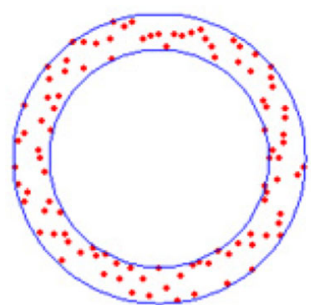

(a)

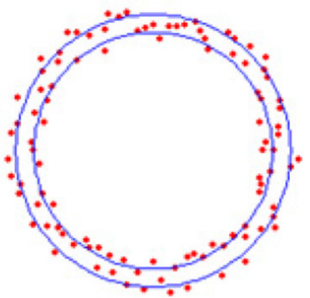

(b) 
We can see the definition of MLS approximation for the case of function approximation from distinct scattered data $[15,16]$. Many variants of MLS have been developed. For example, many strategies occur in specifying bandwidths of the Gauss weight function $[6,22,26]$, the surface used in the models $[4,11,16]$, the weight function $[1,2]$, the fitting criterion $[9,20]$, and so on. Besides, MLS surfaces have been used widely in the last few years. Reference paper [3] uses the MLS surface for point set modeling and rendering. A different point set surface definition in [4] utilizing the critical points of an energy function on lines determined by a vector field is given. This definition reveals connections to research in computer vision and computational topology. Shen et al. [24] describe a method for building interpolating or approximating implicit surfaces from polygonal data and present an improved method for enforcing normal constraints and an iterative procedure for ensuring that the implicit surface tightly encloses their input vertices. MLS has also been successfully applied to image deformation [23], shape optimization [19], animating [17] and many other research areas. The MPA model is set up based on MLS. It resorts to a parabola to approximate a point set from a plane curve or a paraboloid to approximate a point set from a surface. In order to solve the MPA model, the problem is changed to a constrained optimization in a local orthogonal coordinate. The MPA can also be used for adaptive triangular-mesh reconstruction [28]. In this paper, we are persuaded by the MPA approach. However, our approach differs in many respects. We set up a new local model to separate mixed points coming from multiple curves or multiple surfaces. By our algorithm, we will obtain multiple marked points simultaneously in each neighborhood consisting of $k$ nearest neighbors [22] such that all the separated points steer the conventional curve or surface fitting $[8,13]$.

\section{Moving Multiple Curves/Surfaces Approximation}

Moving parabolic approximation [27] as an extension of MLS [16] is powerful for curves and surfaces modeling. Compared to MLS, which is first-order projection, MPA is a model of second-order projection. Given a dataset $P$ and a point $X \in R^{3}$ near the underlying surface $S$. Let $o_{x}$ be the foot point of $X$ on $S$, where $\mathbf{n}$ is the unit normal vector to $S$ at $o_{x}$. Let $\{\mathbf{u}(\mathbf{n}), \mathbf{v}(\mathbf{n})\}$ be the perpendicular unit basis vectors of the tangent plane such that $\left\{o_{x} ; \mathbf{u}(\mathbf{n}), \mathbf{v}(\mathbf{n}), \mathbf{n}\right\}$ forms a local orthogonal system. An MPA model for a surface can be set up accordingly:

$$
\min \sum_{p \in P}\left[h_{p}-\frac{1}{2}\left(a u_{p}^{2}+2 b u_{p} v_{p}+c v_{p}^{2}\right)\right]^{2} \theta\left(\left\|p-o_{x}\right\|\right)
$$

where $\left(u_{p}, v_{p}, h_{p}\right)$ is the coordinate of $p$ in the local orthogonal coordinate system, and $\theta\left(\left\|p-o_{x}\right\|\right)$ is a weight function. It is usually changed to a constrained optimization model to find $o_{x}$ which is on the underlying surface and we can get the paraboloid approximation $h(u, v)=\frac{1}{2}\left(a u^{2}+2 b u v+c v^{2}\right)$ of $P$. Simultaneously, the differential quantities at $o_{x}$ can be computed. Our approach is mainly motivated by [27], and we establish a local model called MMC/SA. There is only one curve or one surface in an MPA model. Compared with MPA, in an MMC/SA model, we use multiple curves 


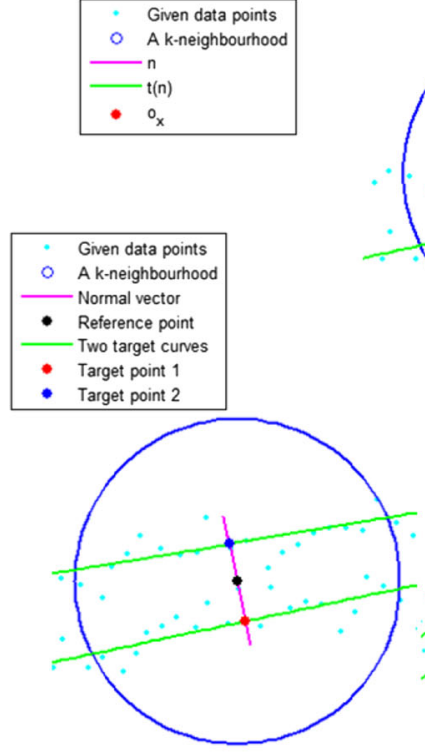

(b)

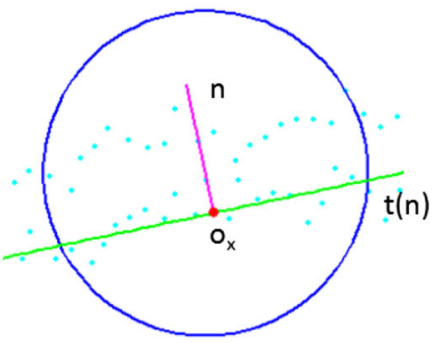

(a)

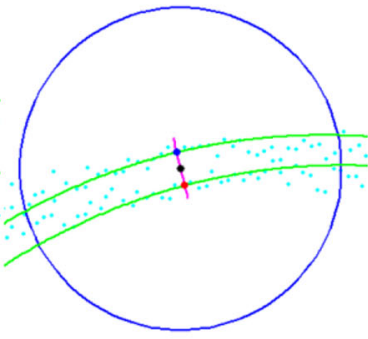

(c)

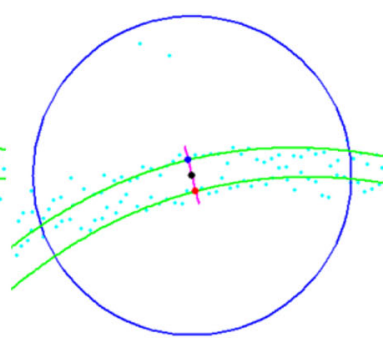

(d)

Fig. 2 Local information display: a the local orthogonal coordinate system for two straight lines approximation; $\mathbf{b}$ the local information of two straight lines approximation; $\mathbf{c}$ the local information of two parabolas approximation; $\mathbf{d}$ the local information of two parabolas approximation with outliers

or multiple surfaces. Our model can also be converted to a constrained optimization, and by MMC/SA algorithm, we can obtain as many points as the number of curves or surfaces in each model to express local underlying positions.

\subsection{MMC/SA Models}

Denote $\left\{Q_{i}\right\}_{i=1}^{n}$ as the scanning or sampling points from two or more underlying curves or surfaces. $X$ is a fixed point called a reference point near the underlying shape, see Figs. $3 \mathrm{c}$ and 6 . We can find its neighborhood $B_{x}$ containing $k$ points $\left\{P_{i}\right\}_{i=1}^{k} \subseteq\left\{Q_{i}\right\}_{i=1}^{n}$ nearest to $X . B_{x}$ is a circle in $2 \mathrm{D}$ which is centered at $X$, and the radius is the maximum Euclidean distance from $X$ to $P_{i} \in B_{x}$, see Figs. 2 and 3c. The value of $k$ will be found in the implementation details. Suppose that $o_{x}$ is a projected point of $X$ on an underlying curve or surface. For convenience, throughout this paper, $X, B_{x}$ and $o_{x}$ have the same meaning. Corresponding to MLS and MPA, we provide a local model in each $B_{x}$ as follow:

$$
\min \sum_{i=1}^{k}\left(A_{1}\left(P_{i}\right)\right)^{2}\left(A_{2}\left(P_{i}\right)\right)^{2} \cdots\left(A_{s}\left(P_{i}\right)\right)^{2} \theta\left(\left\|P_{i}-o_{x}\right\|\right),
$$

where, $A_{l}(x)=0, l=1, \cdots, s$ are normalized algebraic curves/surfaces. $s$ is the number of curves/surfaces in a model. $A_{l}\left(P_{i}\right), l=1, \cdots, s$ are the algebraic distances from $P_{i}$ to $A_{l}(x)=0, l=1, \cdots, s$ respectively. $\theta(d)$ is a weight function, which is smooth, 

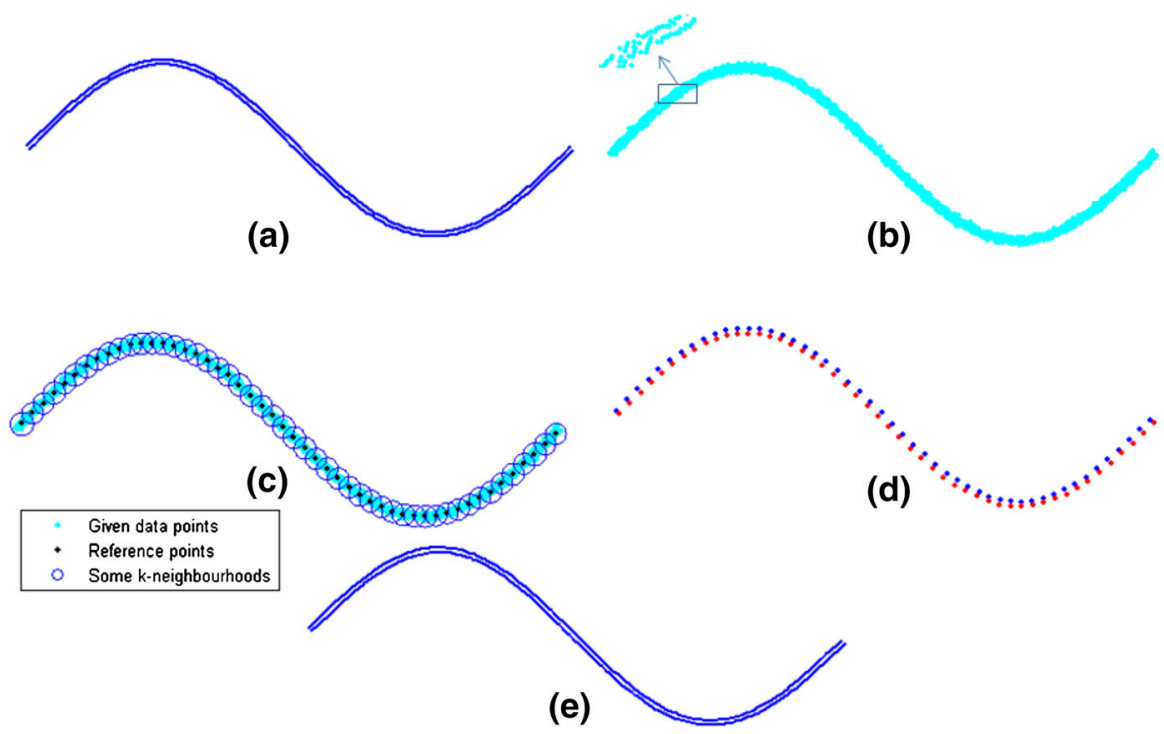

Fig. 3 Sine curve test by two straight lines approximation: a $y=\sin x$ and its offset curve; b The Gauss noise sampling points and a close-up view; c The reference points and their $k$-nearest neighbors; $\mathbf{d}$ The results of MMCA models; e Cubic B-spline curves fitting respectively

monotone decreasing, and positive for any $d \geq 0$. The product of several squared algebraic distance errors appears in the model. Our aim is to minimize the sum of the weighted product errors. As long as one point belongs to an algebraic curves/surfaces in (2), the final result is 0 , so the model (2) is reasonable. However, (2) is highly non-linear which is often synonym of instability and expensive computation. Based on the assumption that $X$ is close to $o_{x}$, we reduce the nonlinearity by simplifying (2) to

$$
\min \sum_{i=1}^{k}\left(A_{1}\left(P_{i}\right)\right)^{2}\left(A_{2}\left(P_{i}\right)\right)^{2} \cdots\left(A_{s}\left(P_{i}\right)\right)^{2} \theta\left(\left\|P_{i}-X\right\|\right)
$$

and find an effective algorithm. The number $s$ and the types of curves/surfaces will be specified by a user according to the shape of point cloud. We aim to find the projected points of $X$ on $A_{l}(x)=0, l=1, \cdots, s$. The projected points are called target points. We call the model (3) MMC/SA instead of model (2) in this paper. It is a local model, and it can be used to separate mixed data received from thin machine parts and patches of broken relics. The curves/surfaces in (3) are called target curves/surfaces. By an appropriate algorithm, we can obtain target points approximating multiple curves/surfaces. The target points are conducive to curve/surface fitting.

To demonstrate the details of the process, we only consider the cases of two curves or two surfaces in a model, i.e., $s=2$. Two straight lines and two parabolas approximation, two planes and two paraboloids approximation are presented based on local maps of differential geometry [7] and analytic representation of curves and surfaces [21]. To guarantee the stability of numerical calculation, in this situation, the weight function is taken as 


$$
\theta(\|P i-X\|)=\frac{e^{-\frac{\|P i-X\|^{2}}{\rho^{2}}}}{\sum_{P_{i} \in B_{x}} e^{-\frac{\|P i-X\|^{2}}{\rho^{2}}}},
$$

denoted as $\theta_{i}$. The parameter $\rho$ in the Gauss weight function is half of the average Euclidean distance between the data points in the $B_{x}$ in our most experiments.

\subsection{D Case: Two Straight Lines and Two Parabolas}

For 2D, we consider the sampling points $\left\{Q_{i}\right\}_{i=1}^{n}$ from two close plane curves $C_{1}$ and $C_{2}$. So the noisy sampling points from $C_{1}$ and $C_{2}$ may mix together, and we cannot distinguish which curve the points belong to, especially for the points along the centerline of $\left\{Q_{i}\right\}_{i=1}^{n}$. Our purpose is to find two target points utilizing an MMCA model established in a $B_{x}$ consisting of $\left\{P_{i}\right\}_{i=1}^{k} \subseteq\left\{Q_{i}\right\}_{i=1}^{n}$. At first, we assume that a reference point $X$ is projected onto $C_{1}$. The foot point is $o_{x} . \mathbf{n}=\left(n_{1}, n_{2}\right)^{\top}$ is the unit vector at $o_{x}$ paralleling with the direction of projection. The unit vector $\mathbf{t}(\mathbf{n})=$ $\left(-n_{2}, n_{1}\right)^{\top}$ is perpendicular to $\mathbf{n}$ such that $\left\{o_{x} ; \mathbf{t}(\mathbf{n}), \mathbf{n}\right\}$ forms a local orthogonal coordinate system, as illustrated in Fig. 2a. So $T_{1}=o_{x}$ can be considered as one target point, and we can use $h=0$ and $h-b t-c=0$ for two straight lines approximation in each $B_{x}$, where $b, c$ are unknown parameters.

Since some quadric curves, such as a hyperbola and an ellipse, can approximate two curves itself, we exclude them and consider a special class of parabolas for two parabolas approximation in the similar local coordinate system. $h-\frac{1}{2} a_{1} t^{2}=0$ and $h-$ $\frac{1}{2} a_{2} t^{2}-b_{2} t-c_{2}=0$ are adopted here, where $a_{1}, a_{2}, b_{2}$ and $c_{2}$ are unknown parameters.

Let $\left(t_{i}, h_{i}\right)$ be the coordinate of $P_{i}$ in the local orthogonal coordinate system, where $t_{i}=\left(P_{i}-o_{x}\right)^{\top} \mathbf{n}$ and $h_{i}=\left(P_{i}-o_{x}\right)^{\top} \mathbf{t}(\mathbf{n})$. Now we first provide the two straight lines model to get the approximation of two close plane curves as follow:

$$
\min \sum_{i=1}^{k} h_{i}^{2}\left(h_{i}-b t_{i}-c\right)^{2} \theta_{i} .
$$

Let $q_{i}=P_{i}-X$ and $o_{x}=X+\xi \mathbf{n} . \mathbf{n}$ is the unit normal at $o_{x} . \xi$ is the signed distance. We change (5) to the following optimization model (6) with a constraint:

$$
\begin{array}{ll}
\min f(\mathbf{n}, \xi, b, c)= & \sum_{i=1}^{k}\left(q_{i}^{\top} \mathbf{n}-\xi\right)^{2}\left(q_{i}^{\top} \mathbf{n}-\xi\right. \\
& \left.-b\left(q_{i}^{\top} \mathbf{t}(\mathbf{n})\right)-c\right)^{2} \theta_{i}, \\
\text { s.t. } \mathbf{n}^{\top} \mathbf{n}=1 . &
\end{array}
$$

In this constrained optimization, $\mathbf{n}, \xi, b$ and $c$ are decision variables. $T_{2}=o_{x}+c \mathbf{n}$, which is the intersection of target curve $h-b t-c=0$ and the coordinate axe decided by the direction of $\mathbf{n}$, can be regarded as another target point, because it is much easier to find than compute projected points. Subsequently, the two target points $T_{1}=o_{x}$ 
and $T_{2}=o_{x}+c \mathbf{n}$ will be used to find the underlying shape. Likewise, we build the two parabolas model for plane curves.

$$
\min \sum_{i=1}^{k}\left(h_{i}-\frac{1}{2} a_{1} t_{i}^{2}\right)^{2}\left(h_{i}-\frac{1}{2} a_{2} t_{i}^{2}-b_{2} t_{i}-c_{2}\right)^{2} \theta_{i} .
$$

Then we change it to an optimization problem as follows:

$$
\begin{aligned}
& \min f\left(\mathbf{n}, \xi, a_{1}, a_{2}, b_{2}, c_{2}\right)=\sum_{i=1}^{k}\left(q_{i}^{\top} \mathbf{n}-\xi-\frac{1}{2} a_{1}\left(q_{i}^{\top} \mathbf{t}(\mathbf{n})\right)^{2}\right)^{2} \\
& \quad \times\left(q_{i}^{\top} \mathbf{n}-\xi-\frac{1}{2} a_{2}\left(q_{i}^{\top} \mathbf{t}(\mathbf{n})\right)^{2}-b_{2}\left(q_{i}^{\top} \mathbf{t}(\mathbf{n})\right)-c_{2}\right)^{2} \theta_{i}, \\
& \text { s.t. } \mathbf{n}^{\top} \mathbf{n}=1,
\end{aligned}
$$

where $\mathbf{n}, \xi, a_{1}, a_{2}, b_{2}$ and $c_{2}$ are also decision variables. Finally, $T_{1}=o_{x}$ and $T_{2}=o_{x}+c_{2} \mathbf{n}$. For two straight lines and two parabolas approximation, we use the same type of curves in a model. However, we can utilize different types such as a straight line and a parabola in a model if necessary and change the algorithm accordingly.

\subsection{D Case: Two Planes and Two Paraboloids}

The ideas from the 2D case can be generalized to a 3D scenario. Let points $\left\{Q_{i}\right\}_{i=1}^{n}$, be sampled from two close underlying surfaces $S_{1}$ and $S_{2} . X$ is a reference point, and $B_{x}$ is the neighborhood of $X$ related to $k$-nearest neighbors $\left\{P_{i}\right\}_{i=1}^{k} \subseteq\left\{Q_{i}\right\}_{i=1}^{n}$ in 3D. Assume that the projection of $X$ is on the underlying surface $S_{1}$, also denoted as $o_{x} . \mathbf{n}$ is the unit normal at $o_{x}$. Let $\{\mathbf{u}(\mathbf{n}), \mathbf{v}(\mathbf{n})\}$ be the perpendicular unit basis vectors of the tangent plane at $o_{x}$ such that $\left\{o_{x} ; \mathbf{u}(\mathbf{n}), \mathbf{v}(\mathbf{n}), \mathbf{n}\right\}$ forms a local orthogonal coordinate system. Thus, $h_{i}=\left(P_{i}-o_{x}\right)^{\top} \mathbf{n}, u_{i}=\left(P_{i}-o_{x}\right)^{\top} \mathbf{u}(\mathbf{n})$, and $v_{i}=\left(P_{i}-o_{x}\right)^{\top} \mathbf{v}(\mathbf{n})$ also hold. So for two planes approximation, $S_{1}$ can be approximated by $h=0$, and $S_{2}$ can be approximated by $h-a u-b v-c=0$ in the local orthogonal coordinate system, where $a, b$ and $c$ are unknown parameters. As a result, the local model of two planes approximation for two close surfaces is

$$
\min \sum_{i=1}^{k} h_{i}^{2}\left(h_{i}-a u_{i}-b v_{i}-c\right)^{2} \theta_{i}
$$

Set $q_{i}=P_{i}-X, o_{x}=X+\xi \mathbf{n}$ and reformulate the model above as a constrained optimization.

$$
\begin{aligned}
& \min f(\mathbf{n}, \xi, a, b, c)=\sum_{i=1}^{k}\left(q_{i}^{\top} \mathbf{n}-\xi\right)^{2}\left[\left(q_{i}^{\top} \mathbf{n}-\xi\right)-\right. \\
& \text { s.t. } \mathbf{n}^{\top} \mathbf{n}=1,
\end{aligned}
$$


where, $\mathbf{n}, \xi, a, b$ and $c$ are decision variables. Let $\mathbf{n}=\left(n_{1}, n_{2}, n_{3}\right)^{\top}$,

$$
\mathbf{u}(\mathbf{n})=\left(\begin{array}{c}
\frac{n_{1}^{2}}{1+n_{3}}-1 \\
\frac{n_{1} n_{2}}{1+n_{3}} \\
n_{1}
\end{array}\right), \quad \mathbf{v}(\mathbf{n})=\left(\begin{array}{c}
\frac{n_{1} n_{2}}{1+n_{3}} \\
\frac{n_{2}^{2}}{1+n_{3}}-1 \\
n_{2}
\end{array}\right)
$$

By a proper initialization of $\mathbf{n}$, we can ensure that $n_{3} \neq-1$. Here $T_{1}=o_{x}$ and $T_{2}=o_{x}+c \mathbf{n}$. In the similar local orthogonal coordinate system, the two paraboloids approximation can use $h-\frac{1}{2}\left(a_{1} u^{2}+2 b_{1} u v+c_{1} v^{2}\right)=0$ and $h-\left(\frac{1}{2}\left(a_{2} u^{2}+2 b_{2} u v+\right.\right.$ $\left.\left.c_{2} v^{2}\right)+d_{2} u+e_{2} v+f_{2}\right)=0$ for surfaces approximation. So the two paraboloids approximation framework in $3 \mathrm{D}$ is

$$
\begin{gathered}
\min \sum_{i=1}^{k}\left[h_{i}-\frac{1}{2}\left(a_{1} u_{i}^{2}+2 b_{1} u_{i} v_{i}+c_{1} v_{i}^{2}\right)\right]^{2}\left[h_{i}-\left(\frac { 1 } { 2 } \left(a_{2} u_{i}^{2}+\right.\right.\right. \\
\left.\left.\left.2 b_{2} u_{i} v_{i}+c_{2} v_{i}^{2}\right)+d_{2} u_{i}+e_{2} v_{i}+f_{2}\right)\right]^{2} \theta_{i}
\end{gathered}
$$

Its constrained optimization model is

$$
\begin{aligned}
\min & f\left(\mathbf{n}, \xi, a_{1}, b_{1}, c_{1}, a_{2}, b_{2}, c_{2}, d_{2}, e_{2}, f_{2}\right)=\sum_{i=1}^{k}\left[\left(q_{i}^{\top} \mathbf{n}-\xi\right)\right. \\
& -\frac{1}{2}\left(a_{1}\left(q_{i}^{\top} \mathbf{u}(\mathbf{n})\right)^{2}+2 b_{1}\left(q_{i}^{\top} \mathbf{u}(\mathbf{n})\right)\left(q_{i}^{\top} \mathbf{v}(\mathbf{n})\right)+c_{1}\right. \\
& \left.\left.\left(q_{i}^{\top} \mathbf{v}(\mathbf{n})\right)^{2}\right)\right]^{2} \times\left[\left(q_{i}^{\top} \mathbf{n}-\xi\right)-\left(\frac { 1 } { 2 } \left(a_{2}\left(q_{i}^{\top} \mathbf{u}(\mathbf{n})\right)^{2}+2 b_{2}\right.\right.\right. \\
& \left.\left(q_{i}^{\top} \mathbf{u}(\mathbf{n})\right)\left(q_{i}^{\top} \mathbf{v}(\mathbf{n})\right)+c_{2}\left(q_{i}^{\top} \mathbf{v}(\mathbf{n})\right)^{2}\right)+d_{2}\left(q_{i}^{\top} \mathbf{u}(\mathbf{n})\right) \\
& \left.\left.+e_{2}\left(q_{i}^{\top} \mathbf{v}(\mathbf{n})\right)+f_{2}\right)\right]^{2} \theta_{i}, \\
\text { s.t. } & \mathbf{n}^{\top} \mathbf{n}=1,
\end{aligned}
$$

where $\mathbf{n}, \xi, a_{1}, b_{1}, c_{1}, a_{2}, b_{2}, c_{2}, d_{2}, e_{2}$ and $f_{2}$ are decision variables. Hence $T_{1}=o_{x}$, and $T_{2}=o_{x}+f_{2} \mathbf{n}$. To solve the four constrained optimization problems above, all the variables need initial guesses.

\section{Implementation}

With respect to implementation, we refer to the choice of reference points, the initialization of optimization algorithm, and other details of MMC/SA with the model of $s=2$.

We describe the details of implementation for the model of two planes approximation here. They can be generalized to other three models mentioned in Sect. 3 similarly. First, we can compute a principal surface [5] (or a principal curve [25]) of $\left\{Q_{i}\right\}_{i=1}^{n}$ and regard the sampling points from it as reference points $\left\{X_{j}\right\}_{j=1}^{m}$. In this way, $\left\{X_{j}\right\}_{j=1}^{m}$ are all distributed uniformly in $\left\{Q_{i}\right\}_{i=1}^{n}$ and near the underlying shape, see Fig. 6. For 


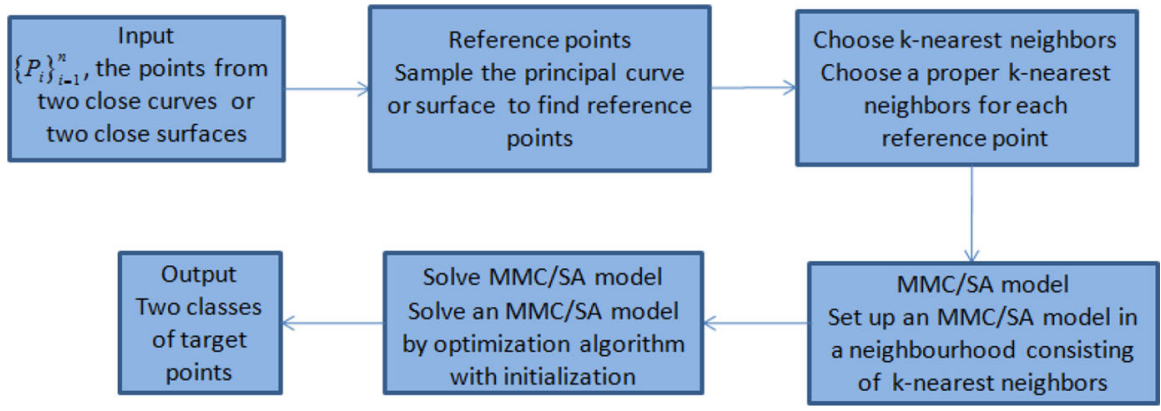

Fig. 4 Implementation pipeline

each reference point $X_{j}$, denoted as $X$, we will find out its $B_{x}$ which must enclose enough points to represent two surfaces. Besides, the value of $k$ in our implementation need to make sure that maximum Euclidean distance from $X$ to $P_{i} \in B_{x}$ is 2.5 times of the local thickness of the point cloud. They are circles in 2D, see Figs. 2 and 3c. The optimization model related to two planes approximation will be solved in the $B_{x}$ using an optimization function 'fmincon' in matlab which is mainly based on sequential quadratic programming (SQP) method [10]. The MMSA algorithm needs initial values for all the parameters. As with the initialization in [27], by first fitting a local hyperplane with norm $\mathbf{n}^{\mathbf{0}}$ according to the data points around $X$, we get the initial value $\mathbf{n}^{\mathbf{0}}$ of $\mathbf{n}$. For two planes approximation, since $S_{1}$ is approximated by $h=0$, and $S_{2}$ is approximated by $h-a u-b v-c=0$, we need the initial guesses $a^{0}, b_{0}$ and $c^{0}$ of $a, b$ and $c$. To get $a^{0}$ and $b_{0}$, we need solve the WLS model below:

$$
\min \sum_{i=1}^{k}\left(\varphi_{i}^{0}-\xi^{0}-\left(a^{0} u_{i}^{0}+b^{0} v_{i}^{0}\right)\right)^{2} \theta_{i}
$$

where $\varphi_{i}^{0}=q_{i}^{\top} \mathbf{n}^{\mathbf{0}}, u_{i}^{0}=q_{i}^{\top} \mathbf{u}\left(\mathbf{n}^{\mathbf{0}}\right), v_{i}^{0}=q_{i}^{\top} \mathbf{v}\left(\mathbf{n}^{\mathbf{0}}\right)$. We can also get the initial values $\xi^{0}$ of $\xi$ according to (13). Lastly, we assume that $x x$ is the uppermost (or the lowest point) point along the direction of $\mathbf{n}^{\mathbf{0}}$ in the $B_{x}$ to keep two different surfaces in an MMSA model not too near. $x x(j)$ represents the $j$ th component of $x x$. Let $h-a^{0} u-b^{0} v-c^{0}=0$ pass through $x x$. The initial guess $c^{0}$ of $c$ is calculated by $c^{0}=$ $x x(3)-a^{0} x x(1)-b^{0} x x(2)$. The initializations for two paraboloids approximation, even for two straight lines approximation and two parabolas approximation, are similar to two planes approximation.

By the MMSA algorithm, we will compute two planes (the case of two planes) approximating the underlying shape in a $B_{x}$. The details of obtaining two target points are shown in Sect. 3. The similar local information in 2D can be seen in Fig. 2. For all the reference points $\left\{X_{j}\right\}_{j=1}^{m},\left\{Q_{i}\right\}_{i=1}^{n}$ are changed to two classes of target points. Generally speaking, it is a re-sampling process. We expect that the two classes of points can portray the underlying shape. Finally, we do surface fitting [13]. Figure 3 shows the whole process of implementation in 2D. Obviously, the number of the target points fixed by the number of the reference points decides the quality of the result of the surface fitting. In general, the more target points, the better we can express the 


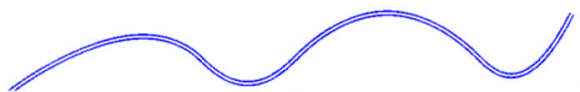

(a)

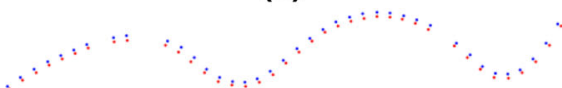

(c)

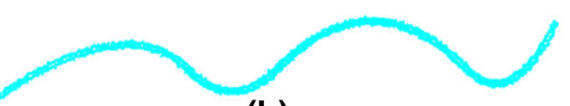

(b)

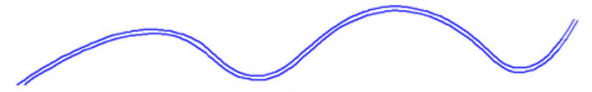

(d)

Fig. 5 Quadratic B-spline curve test by two parabolas approximation: a a quadratic B-spline curve and its offset curve $\mathbf{b}$ the Gauss noise sampling points; $\mathbf{c}$ the results of MMCA models; $\mathbf{d}$ cubic B-spline curves fitting respectively

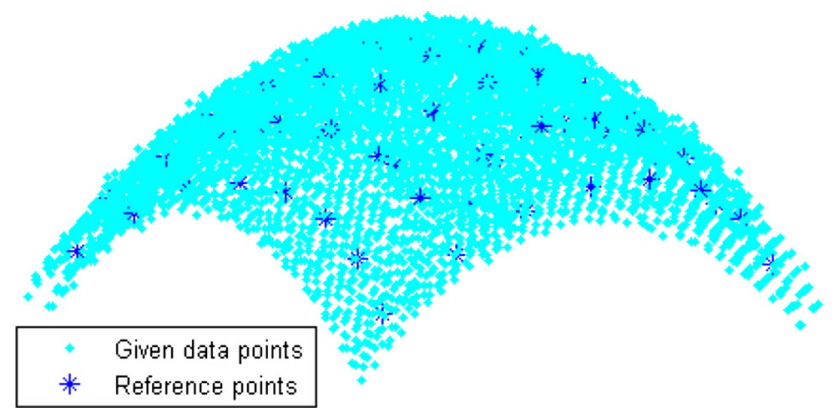

Fig. 6 Reference points obtained by sampling a principal surface

Fig. 7 Complexity data test by four parabolas approximation: a the noisy sampling points from four curves; $\mathbf{b}$ the results of multiple MMCA models

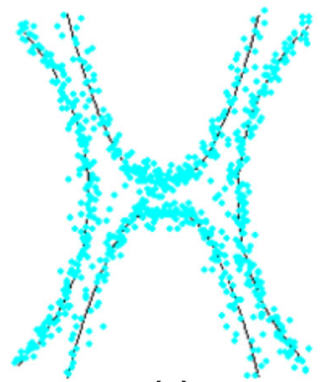

(a)

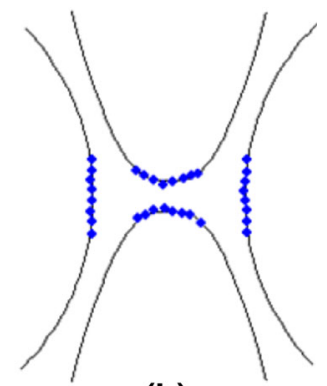

(b)

target shape. An increased amount of reference points, however, increases the amount of models we need to solve. Since efficiency requires equilibrium, we only divide the domain into 64 parts in all the examples in the cases of surfaces. Our implementation pipeline is also illustrated in Fig. 4.

As for space curves, our model framework can also work utilizing three steps in a $B_{x}$. First, find a plane by fitting a local plane according to the data points around the reference point $X$ and establish a local orthogonal coordinate system. Second, project the points in the $B_{x}$ onto the plane above. Third, separate the projected points on the plane by two straight lines or two parabolas approximation according to $2 \mathrm{D}$ case in Sect. 3. The two computed points are regarded as target points. Figure 8 shows the results of applying MMCA models to noisy sampling points from two close space curves. 


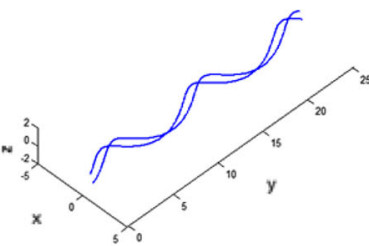

(a)

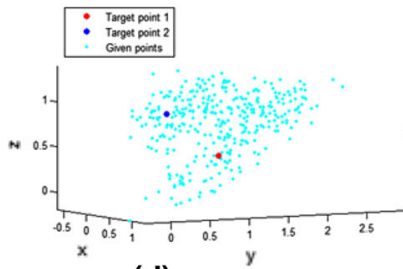

(d)

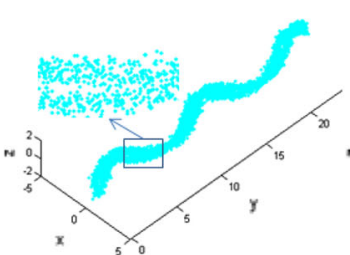

(b)

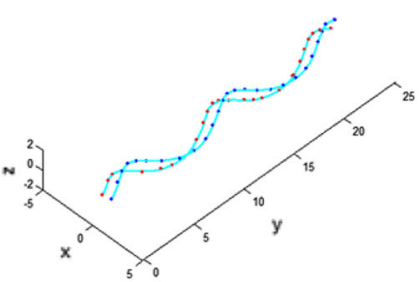

(c)

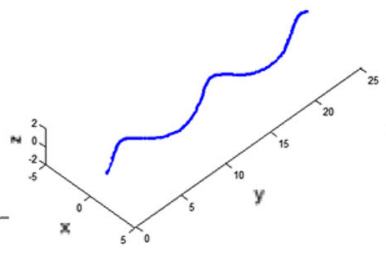

(e)

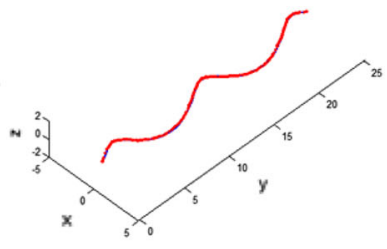

(f)

Fig. 8 Double helix test by two parabolas approximation: a the exact double helix; b the Gauss noise sampling points and a close-up view; $\mathbf{c}$ the results of multiple MMCA models and curves fitting; $\mathbf{d}$ a closeup view of the result about one MMCA model; $\mathbf{e}$ and $\mathbf{f}$ are the results of multiple MMCA models and curves fitting expressed separately

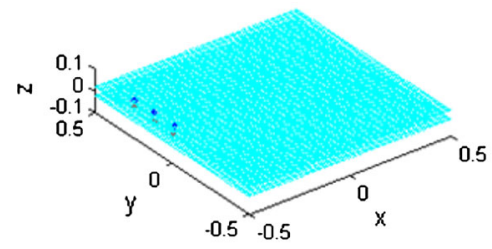

(a)

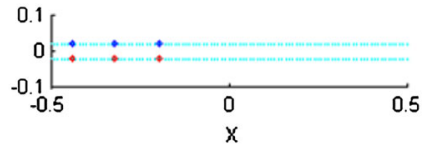

(c)

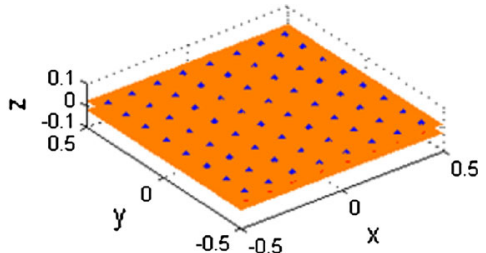

(b)

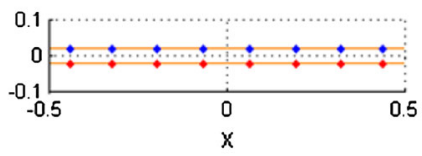

(d)

Fig. 9 Plane test with clean data by two planes approximation: a the sampling points from two exact planes and the results of three MMSA models; $\mathbf{b}$ the results of 64 MMSA models and surfaces fitting; $\mathbf{c}$ a different view of graph (a); d a different view of graph (b)

\section{Examples and Discussions}

Some examples corresponding to Sect. 3 and some limitations about our algorithm are presented in this part.

\subsection{Examples}

In $2 \mathrm{D}$, the result using two straight lines approximation is Fig. 3. Figure $2 \mathrm{~d}$ shows that the MMCA model is insensitive to outliers due to the adjustment of the parameter $\rho$ 


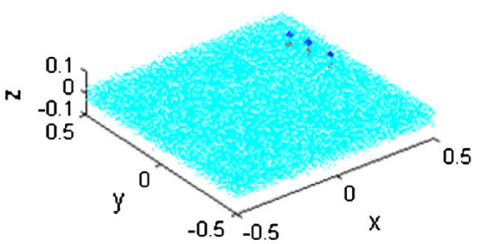

(a)

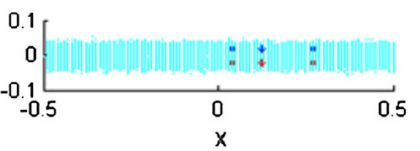

(c)

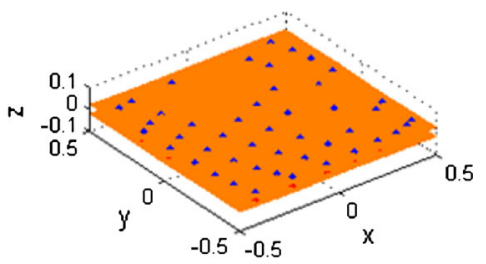

(b)

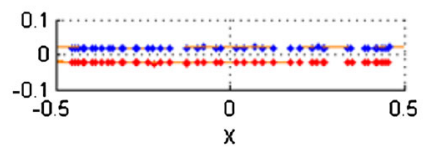

(d)

Fig. 10 Plane test with $22.5 \%$ noise by two planes approximation: a the sampling points with $22.5 \%$ noise from the two planes depicted in Fig. 9a and the results of three MMSA models. $\mathbf{b}$ the result of 64 MMSA models and surfaces fitting; $\mathbf{c}$ a different view of graph (a); d a different view of graph (b)

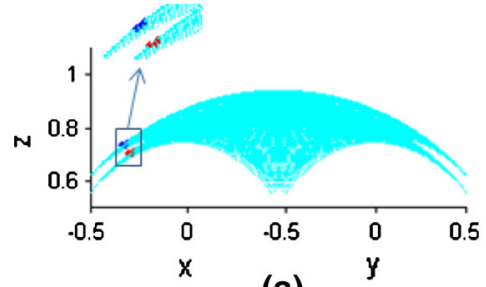

(a)

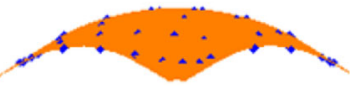

(c)

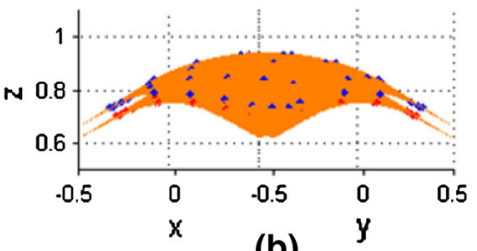

(b)

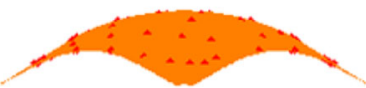

(d)

Fig. 11 Sphere test with clean data by two planes approximation: a the sampling points from the exact sphere and its offset surface and a close-up view of the results about three MMSA models; $\mathbf{b}$ the results of 64 MMSA models and surfaces fitting; $\mathbf{c}$ and $\mathbf{d}$ are the separate representations of (b)

of Gauss weight function. Two parabolas approximation is provided in Fig. 5. In the case of two curves approximation, we usually establish a local coordinate system, and we use two curves to approximate. When the sampling points are acquired from many curves/surfaces, $s>2$ occurs in an MMC/SA model. For example, in Fig. 7, we use four different parabolas to approximate. We did not set up a local coordinate system, and the projected locations of reference points onto four parabolas are regarded as four target points in this situation. Sometimes the optimization algorithm does not converge when the objective function is solved using the $k$-nearest neighbors in some $B_{x}$. But the result is still good by changing the Gauss weight function of objective function to $\frac{1}{\left\|P_{i}-X\right\|^{2}}$. In 3D, the MMCA result of space curves is shown in Fig. 8. We also do some experiments on surfaces and present RMS errs estimation: 

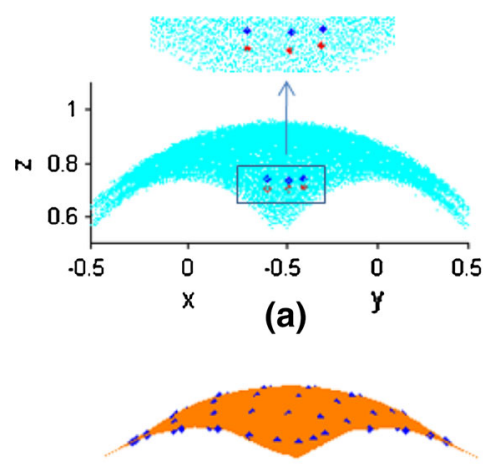

(c)
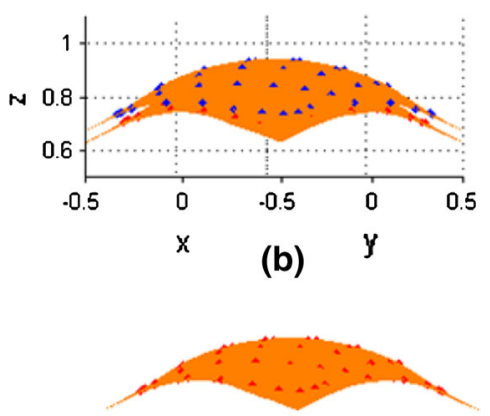

(d)

Fig. 12 Sphere test with $22.5 \%$ noise by two planes approximation: a the sampling points with $22.5 \%$ noise from the surfaces depicted in Fig. 11a and a close-up view of the results about three MMSA models; $\mathbf{b}$ the results of 64 MMSA models and surfaces fitting; $\mathbf{c}$ and $\mathbf{d}$ are the separate representations of (b)

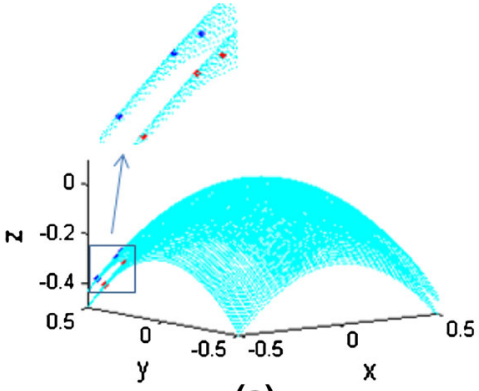

(a)

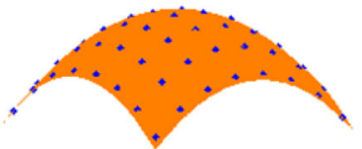

(c)

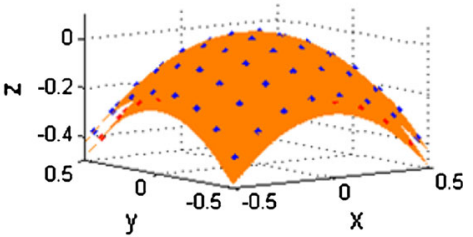

(b)

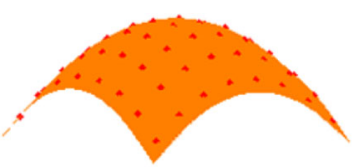

(d)

Fig. 13 Elliptic paraboloid test with clean data by two paraboloids approximation: a the sampling points from the exact elliptic paraboloids its offset surface and a close-up view of the results about three MMSA models; $\mathbf{b}$ the results of 64 MMSA models and surfaces fitting; $\mathbf{c}$ and $\mathbf{d}$ are the separate representations of (b)

$$
e r r=\sqrt{\frac{\sum_{i=1}^{m}\left(d_{i 1}^{2}+d_{i 2}^{2}\right)}{m}}
$$

$d_{i 1}$ represents the minimal distance from the upper target point to the upper exact surface and $d_{i 2}$ represents the minimal distance from the lower target point to the lower exact surface in the ith $B_{x}$.

In order to verify the algorithm, we generate a point cloud by adding Gaussian noise with a magnitude of $22.5 \%$ of the distance between the exact surface and 


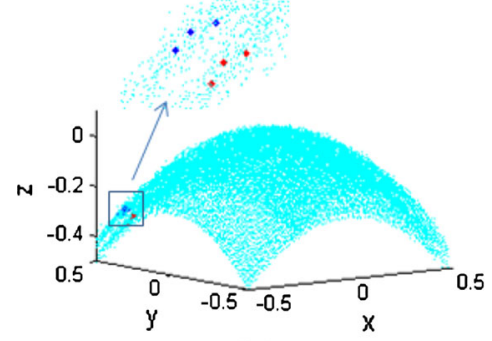

(a)

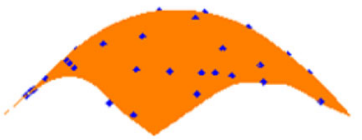

(c)

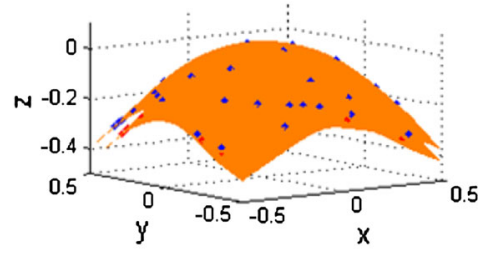

(b)

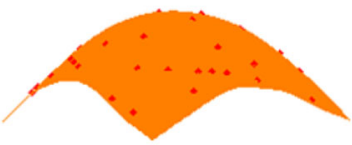

(d)

Fig. 14 Elliptic paraboloid test with $22.5 \%$ noise by two paraboloids approximation: a the sampling points with $22.5 \%$ noise from the surfaces depicted in Fig. 13a and a close-up view of the results about three MMSA models; $\mathbf{b}$ the results of 64 MMSA models and surfaces fitting; $\mathbf{c}$ and $\mathbf{d}$ are the separate representations of (b)

Fig. 15 Hyperboloid paraboloid test with clean data by two paraboloids approximation: a the sampling points from the exact hyperboloid paraboloids and its offset surface and a close-up view of the results about three MMSA models; $\mathbf{b}$ the results of 64 MMSA models and surfaces fitting; $\mathbf{c}$ and $\mathbf{d}$ are the separate representations of (b)

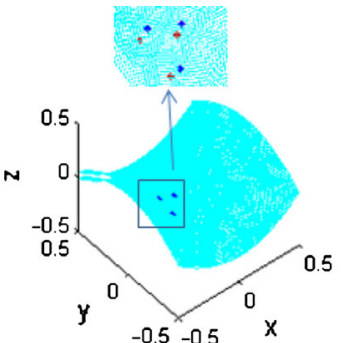

(a)

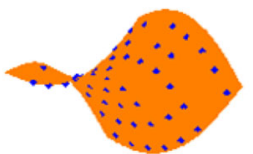

(c)

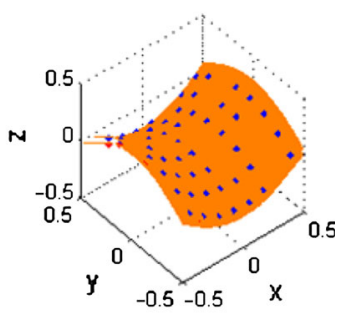

(b)

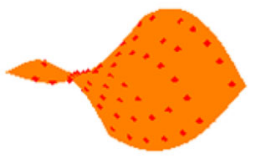

(d)

its offset surface. The test surfaces are related to a plane $z=0.02$, a sphere $z=\sqrt{0.9^{2}-x^{2}-y^{2}}$, a elliptic paraboloid $z=-x^{2}-y^{2}$ and a hyperboloid paraboloids $z=x^{2}-y^{2}$. We consider the bivariate function $z(x, y),(x, y) \in$ $[-0.5,0.5] \times[-0.5,0.5]$, and 64 local MMSA models are solved in each test. Plane test and sphere test using two planes approximation are presented in Figs. 9-12, and elliptic paraboloid test and hyperbolic paraboloid test are displayed in Figs. 13-16. Since partial cover happens in the case of surfaces, the MMSA results are shown in different ways. The error analysis is shown in Tables 1 and 2. Figure 17 shows that our model and algorithm can be applied to non-uniform situations. We also present the MMSA results of the actual data from the inner and outer walls of a part of cylindrical 
Fig. 16 Hyperboloid paraboloid test with $22.5 \%$ noise by two paraboloids approximation: a the sampling points with $22.5 \%$ from the surfaces depicted in Fig. 15a and a close-up view of the results about three MMSA models; $\mathbf{b}$ the results of 64 MMSA models and surfaces fitting; $\mathbf{c}$ and $\mathbf{d}$ are the separate representations of $\mathbf{b}$

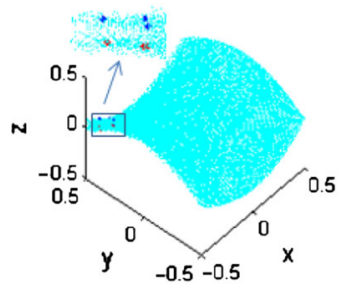

(a)

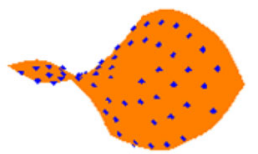

(c)

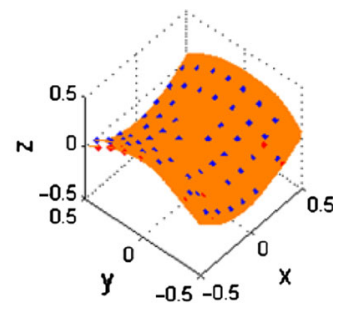

(b)

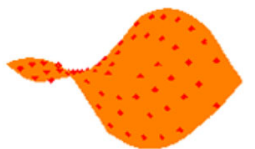

(d)
Table 1 Errs estimation for two planes approximation

\begin{tabular}{ll}
\hline Examples for two planes case & errs \\
\hline Plane (clean data) & $6.3738 \mathrm{e}-007$ \\
Plane (with $22.5 \%$ noise) & $8.9128 \mathrm{e}-004$ \\
Sphere (clean data) & $9.6911 \mathrm{e}-004$ \\
Sphere (with $22.5 \%$ noise) & 0.0039 \\
\hline
\end{tabular}

\begin{tabular}{ll}
\hline Examples for two paraboloids case & errs \\
\hline Elliptical paraboloid (clean data) & $1.1033 \mathrm{e}-004$ \\
Elliptical paraboloid (22.5\% noise) & 0.0051 \\
hyperbolic paraboloid (clean data) & $1.0736 \mathrm{e}-004$ \\
hyperbolic paraboloid (22.5\% noise) & 0.0062 \\
\hline
\end{tabular}

Table 2 Errs estimation for two paraboloids approximation

object by a structured light scanner in Fig. 18. The comparisons with MPA plus surface fitting, boundary approximation, and our MMSA plus surface fitting are also carried out in Fig. 19 to prove the effectiveness of our strategy.

\subsection{Discussions}

It should be noted that the MMC/SA model and the corresponding algorithm presented in the paper just work in a local fashion. The number and types of curves or surfaces in a model can be chosen by users according to the point cloud. If a user deals with a point cloud data from a whole thin-wall object, she/he is expected to use our model and algorithm to deal with the most parts of the data. For the other parts, data from the object part with more complicated topological structure and sharp features, she/he needs to utilize other techniques to finish data separation. 


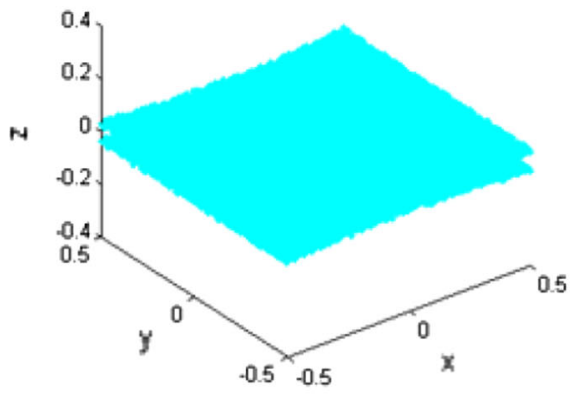

(a)

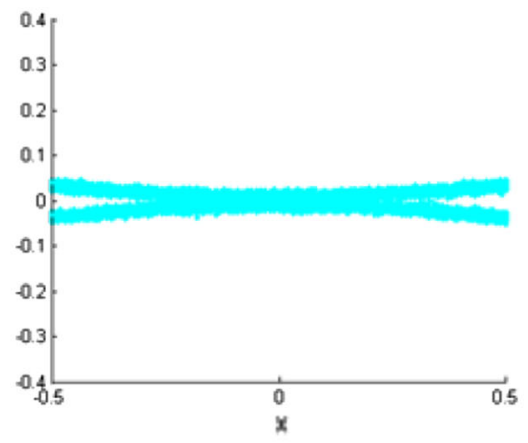

(c)

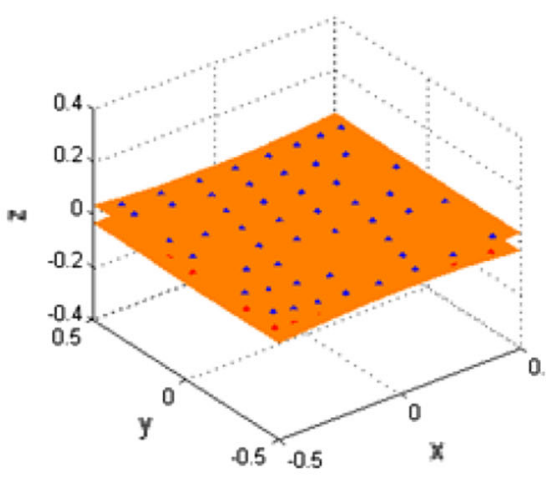

(b)

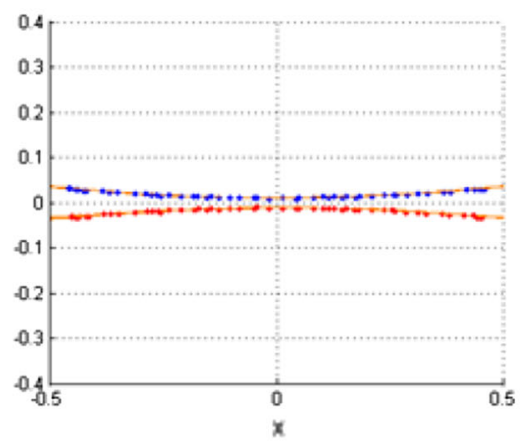

(d)

Fig. 17 Parabolic cylinder test by two paraboloids approximation: a the Gauss noise sampling points from the exact parabolic cylinders; $\mathbf{b}$ the results of 64 MMSA models and surfaces fitting; $\mathbf{c}$ a different view of graph (a); d a different view of graph (b)

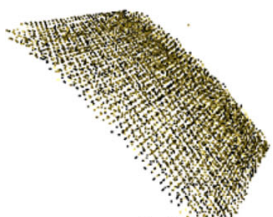

(a)

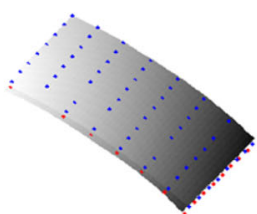

(b)

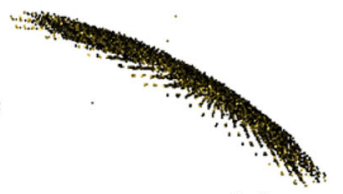

(c)

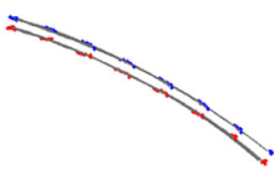

(d)

Fig. 18 Actual data test by two planes approximation: a the scanned points from the inner and outer walls of a part of cylindrical object; $\mathbf{b}$ the results of 64 MMSA models and surfaces fitting; $\mathbf{c}$ a different view of graph (a); d a different view of graph (b)

On the other hand, in the current implementation of MMC/SA model, for each reference point, a single optimization model may consume a few seconds. In the future, we need to consider how to improve the efficiency of the implementation. 


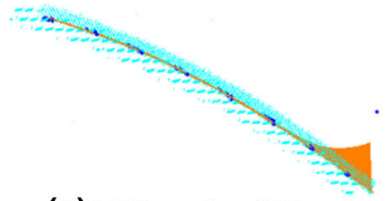

(a) MPA+surface fitting

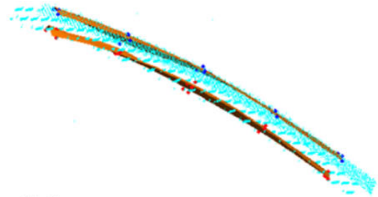

(b) Boundary approximation

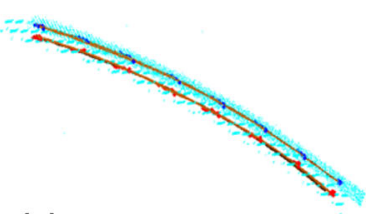

(c) MMSA+surface fitting

Fig. 19 Surface fitting of the real data in Fig. 18 with different techniques. a MPA plus surface fitting; b boundary approximation similar to [29]; b our MMSA plus surface fitting

\section{Conclusion}

A local model called MMC/SA to separate mixed points from multiple curves or surfaces have now been provided. The number and types of curves or surfaces in a model can be chosen by users themselves according to the point cloud, and it is insensitive to outliers. In this paper, we focus on the case of two curves or two surfaces in a model. Two straight lines and two parabolas approximation, two planes and two paraboloids approximation are elaborated. We have tested our algorithm on many examples including various curves (including plane curves and space curves) and surfaces to prove its effectiveness combined with the requisite error analysis. A simple example test using four parabolas proves that our model can be extended to more curves/surfaces approximation. Real data from a part of cylindrical object by a structured light scanner are also tested. We emphasize that the model is a local model. The comparisons with MPA plus surface fitting, boundary approximation, and our MMSA plus surface fitting are also carried out to prove the effectiveness of our strategy. We expect that our MMC/SA method will be used in reverse engineering application, such as modeling of thin machine parts and patches of broken relics. Nevertheless, our current algorithm has several limitations that we wish to address in our future work. First, we need to reduce time consumption. Second, if the algorithm is improved in adaptively changing the type of surfaces and detecting the number of different surfaces automatically, it will be better.

Acknowledgments The authors are supported by the 111 Project (No. b07033), the NSF of China (11031007, 11371341 and 11171332), and Program for New Century Excellent Talents in University (No. NCET-11-0881).

\section{References}

1. Adamson, A., Alexa, M.: Anisotropic point set surfaces. In: Computer Graphics Forum, vol. 25, pp. 717-724. Wiley Online Library (2006)

2. Alexa, M., Adamson, A.: Interpolatory point set surfaces-convexity and Hermite data. ACM Trans. Gr. (TOG) 28(2), 20 (2009)

3. Alexa, M., Behr, J., Cohen-Or, D., Fleishman, S., Levin, D., Silva, C.T.: Computing and rendering point set surfaces. Vis. Comput. Gr. IEEE Trans. 9(1), 3-15 (2003)

4. Amenta, N., Kil, Y.J.: Defining point-set surfaces. ACM Trans. Gr. (TOG) 23(3), 264-270 (2004)

5. Bishop, C.M., Svensén, M., Williams, C.K.: Gtm: the generative topographic mapping. Neural comput. 10(1), 215-234 (1998)

6. Dey, T.K., Sun, J.: An adaptive mls surface for reconstruction with guarantees. In: Symposium on Geometry Processing, pp. 43-52. Vienna (2005) 
7. do Carmo, M.P.: Differential Geometry of Curves and Surfaces. Prentice-Hall, Englewood Cliffs (1976)

8. Farin, G.E.: Curves and Surfaces for CAGD: A Practical Guide. Morgan Kaufmann, Burlington (2002)

9. Fleishman, S., Cohen-Or, D., Silva, C.T.: Robust moving least-squares fitting with sharp features. ACM Trans. Gr. (TOG) 24, 544-552 (2005)

10. Gilbert, J.C., Lemaréchal, C.: Numerical Optimization: Theoretical and Practical Aspects. Springer, Heidelberg (2006)

11. Guennebaud, G., Gross, M.: Algebraic point set surfaces. CM Trans. Gr. (TOG) 26, 23 (2007)

12. Kanaya, I., Chen, Q., Kanemoto, Y., Chihara, K.: Threedimensional modeling for virtual relic restoration. Multimed. IEEE 7(2), 42-44 (2000)

13. Keim, D.A., Herrmann, A.: The gridfit algorithm: an efficient and effective approach to visualizing large amounts of spatial data. In: Proceedings of the conference on Visualization'98, pp. 181-188. IEEE, Research Triangle Park (1998)

14. Lancaster, P., Salkauskas, K.: Surfaces generated by moving least squares methods. Math. Comput. 37(155), 141-158 (1981)

15. Levin, D.: The approximation power of moving least-squares. Math. Comput. Am. Math. Soc. 67(224), 1517-1531 (1998)

16. Levin, D.: Mesh-independent surface interpolation. Geometric Modeling for Scientific Visualization, pp. 37-49. Springer, Heidelberg (2004)

17. Müller, M., Keiser, R., Nealen, A., Pauly, M., Gross, M., Alexa, M.: Point based animation of elastic, plastic and melting objects. In: Proceedings of the 2004 ACM SIGGRAPH/Eurographics symposium on computer animation, pp. 141-151. Eurographics Association, Grenoble (2004)

18. Nealen, A.: An as-short-as-possible introduction to the least squares, weighted least squares and moving least squares methods for scattered data approximation and interpolation. URL: http://www.nealen. com/projects, 130, 150 (2004)

19. Oudjene, M., Ben-Ayed, L., Delameziere, A., Batoz, J.-L.: Shape optimization of clinching tools using the response surface methodology with moving least-square approximation. J. Mater. Process. Technol. 209(1), 289-296 (2009)

20. $\ddot{O}$ ztireli, A.C., Guennebaud, G., Gross, M.: Feature preserving point set surfaces based on non-linear kernel regression. In: Computer Graphics Forum, vol. 28, pp. 493-501. Wiley Online Library (2009)

21. Patrikalakis, N.M., Maekawa, T.: Shape Interrogation for Computer Aided Design and Manufacturing. Springer, Heidelberg (2002)

22. Pauly, M.: Point primitives for interactive modeling and processing of 3D geometry. Citeseer (2003)

23. Schaefer, S., McPhail, T., Warren, J.: Image deformation using moving least squares. ACM Trans. Gr. (TOG) 25, 533-540 (2006)

24. Shen, C., O'Brien, J.F., Shewchuk, J.R.: Interpolating and approximating implicit surfaces from polygon soup. ACM Trans. Gr. (TOG) 23, 896-904 (2004)

25. Verbeek, J.J., Vlassis, N., Kröse, B.: A k-segments algorithm for finding principal curves. Pattern Recognit. Lett. 23(8), 1009-1017 (2002)

26. Wang, H., Scheidegger, C.E., Silva, C.T.: Optimal bandwidth selection for mls surfaces. In: Shape Modeling and Applications, 2008. IEEE International Conference on SMI 2008, pp. 111-120. IEEE (2008)

27. Yang, Z., Kim, T.-W.: Moving parabolic approximation of point clouds. Computer-Aided Des. 39(12), 1091-1112 (2007)

28. Yang, Z., Seo, Y.-H., Kim, T.-W.: Adaptive triangular-mesh reconstruction by mean-curvature-based refinement from point clouds using a moving parabolic approximation. Computer-Aided Des. 42(1), 2-17 (2010)

29. Flöry, S.: Fitting curves and surfaces to point clouds in the presence of obstacles. Comput. Aided Geom. Des. 26(2), 192-202 (2009) 